\title{
Recherches expérimentales sur la stabilité d'une jetée à talus incliné soumise à la houle
}

\section{Experimental research on the stability of a rubble mound breakwater subjected to wave action}

\author{
pAR R. BARBE ET C. BEAUDEVIN \\ INGÉNIEURS AU LABORATOIRE DAEPHINOIS D'HYDRAULIQUE
}

\begin{abstract}
Dans le cadre de ses éludes générales sur modèles réduits, le. Laboratoire Dauphinois d'Hydraulique a examiné d'une façon systématique la stabilité à la houle. de jefées à talus avec carapaces en enrochements on en blocs en vrac. Après une étude qualilative de la constitution d'un tel ouvrage par jet d'enrochements dans la houle, on a examiné rapidement l'influence de chacun des paramètres régissant la stabilité d'une jetée d̀ talus. Un abaque général condense les résultats obtenus à ce jour et permet des calculs d'avant-projet.
\end{abstract}

\begin{abstract}
The "Laboraloire Dauphinois d'Hydraulique" has examined in a systematic manner, within the limits of its general studies on scale models, the slability of rubble mound break waters with carapaces of rough stone or loose blocks.

Following a aualilative. study of the achievement of such an installation by dumping rough rocks into the waves, the influence of each of the parameters governing the stabilit!l of the rubble mound breakwater has been anickly examined. A general graph condenses the results obtrined up to now and enables the calculations for the preliminary plan.
\end{abstract}

\section{I. - POSITION DU PROBLÈME}

\section{Stabilité des jetées à talus}

Le problème de la stabilité à la mer d'une jetée a toujours, à juste titre, retenu l'attention des ingénieurs.

Définir un ouvrage économique et susceptible cependant de résister, sans dommages trop importants, aux plus violentes houles auxquelles il nuisse être soumis, est en effet une cuestion difficile à résoudre. La stabilité des différentes phases de construction doit en effet être également assurée, sinon pour les plus violentes houles que l'on peut craindre, tout au moins pour des tempêtes courantes.
Les jetées que l'on peut être amené à construire se rattachent toutes, en première apnroximation, à deux classes d'ouvrages: les dianes verticales et les diques à talus, ces dernières nossédant une carapace côté large formée soit de blocs artificiels arrimés, soit d'enrochements ou de bloes artificiels en vrac.

Mais si leur raison d'être reste en définitive la même - empêcher l'agitation de la mer d'atteindre les régions au'elles sont censées protéger - leurs modes d'action sont essentiellement différents. En effé, tandis que sur les digues verticales l'énergie de la houle incidente se réfléchit presque totalement, sur une digue à talus, 
au contraire, elle se dissipe particllement en un déferlement tumultueux $\left(^{*}\right)$.

S'il semble historiquement que, dans l'antiquité, les digues verticales furent les premières à être construites sur une grande échelle ${ }^{\star \star}$ ), la technique s'en perdit bientôt et les digues à talus devinrent et restèrent d'emploi courant. Plus ou moins importantes suivant les besoins locaux et suivant les moyens mis en cuvre, de telles jetées ont été construites partout; mais sur leur principe même, comme en beaucoup d'autres domaines de la technique; on ne savait encore que peu de choses vers le début du siècle.

On se bornait en général à construire des ouvrages toujours plus puissants que ceux connus qui, placés dans des situations analogues, n'avaient pas résisté à la violence d'un coup de mer. Nous n'avons nullement l'intention de critiquer un tel état de fait, car l'Ingénieur a toujours été placé devant l'absolue nécessité de construire des ouvrages qu'il était, par ailleurs, impossible de calculer, même approximativement.

C'est pourquoi on comprend l'attrait qu'inspira la digue verticale, lorsque les travaux célèbres d'une pléiade d'esprits éminents, notamment Boussineso, Sainflou, Benezit, Gourret et Watrer, pour ne citer que quelques-uns des disparus, permirent, par une solution de plus en plus exacte du clapotis, de calculer les efforts périodiques qui sollicitent un tel ouvrage. Toutefois, cette disposition conduit souvent à une réalisation délicate et elle n'est pas d'un emploi général, notamment dans les régions où le marnage est important et lorsqu'on veut éviter autant que possible tout ressac. On se trouve alors ramené à la jetée $\dot{a}$ talus, dont le jroblème est resté presque neuf, malgré les progres immenses accomplis dans le domaine de l'hydrodynamique des vagues et malgré la puissance énorme des moyens en personnel et en matériel que mettent maintenant en jeu les entreprises de travaux maritimes.

C'est que la complexité des phénomenes qui se produisent lors du déferlement de la houle sur une jetée à talus se prête mal à une mise en équation. Si quelques-unes des questions qui se posent, notamment celle de la réflexion et celle du déferlement, sur talus inclinés, ont déjà étó

(*) Nous n'aborderons pas ici le problème des digues mixtes qui, par leur conception, rappellent les digues verticales mais peuvent. en raison de lenr implantation par fonds faibles ou de la cote trop haute de leur soubassement, être soumises, pour certaines conditions de niveau et de houle, au choc des lames déferlantes. Ceci conduit à éviter de plus en plus l'emploi de ce type d'ouvrage.

$\left(^{*}\right)$ Nous n'en citerons pour exemple que les puissantes « antemurales $\$$ romaines des ports de Claude et d'Antonin. abordées mathématiquement par des schémas simples, aucune n'est encore résolue à l'heure actuelle de façon satisfaisante et certaines ne sont qu'à peine ébauchées, tandis que d'autres restent absolument hermétiques à tout calcul.

\section{Emploi du modèle réduit}

Heureusement, là comme en beaucoup d'autres domaines de l'hydrodynamique appliquée, le modèle réduit judicieusement conç peut être d'un grand secours pour l'Ingénieur et il a déis été utilisé souvent à cet effet. Cependant, les dimensions nécessairement limitées d'un tel modèle restaient un obstacle grave à toute recherche sérieuse, puisque la réflexion propre de l'ouvrage à étudier atteignant l'appareil générateur de houle pouvait, en se réfléchissant sur lui, se superposer à la houle incidente et que, par suite, loin d'observer la tenue de l'ouvrage devant une houle pure, on ne pouvait observer que son évolution sous l'effet d'un clapotis complexe plus ou moins grossier. Un progrès essentiel a été réalisé dans la technique de ces modèles réduits par l'emploi conjugué du filtre a houle (*) et des absorbeurs d'énergie judicieusement placés, qui permettent de produire des houles de caractéristiques parfaitement définies et facilement reproductibles.

Sous réserve de préciser par une campagne d'observations sur place la houle la plus dangereuse qu'il faut craindre pour une phase temporaire ou définitive de l'ouvrage, l'expérience permet facilement, soit de contrôler si un profil préalablement défini est stable, soit même de déterminer a priori le profil stable le plus économique. Dans les deux cas, l'observation commode que permet le modèle réduit peut entrainer par.fois des économies substantielles, en réduisant notamment l'échantillonnage des enrochements ou des blocs artificiels dans les parties de l'ouvrage où la stabilité est surabondante. Il va sans dire toutefois que la plus grande prudence reste de règle dans une telle étude et que, si l'Ingénieur du Laboratoire d'Essai peut préciser expérimentalement les limites de stabilité sur sa maquette, il ne doit cependant formuler ses conclusions qu'en accord étroit avec l'Ingénieur spécialiste de Travaux Maritimes qui a seul l'habitude pratique de telles réalisations en vraic grandeur.

C'est dans cet esprit qne nous présentons ici les résultats des deux séries d'études expérimentales, effectuées au Laboratoire Dauphinois d' $\mathrm{Hy}$ draulique sur le profil d'équilibre des jetées à talus incliné soumises à la hoúle, pensant que de

(*) F. Burser. - Filtre à houle. La Hozille Blanche, $n^{\circ} 3$ de mai-juin 1948 . 
telles recherches pourraient être utiles à tous ceux qui, dans le monde, ont à tracer ou à construire des ouvrages maritimes.

Nos études ont porté sur des digues a no... ments inclinés, composés d'éléments en vrac (blocs artificiels ou enrochements). Nous avons soumis ces ouvrages à l'action de houles qui les attaquaient perpendiculairement.

Nous avons cherché tout d'abord à observer qualitativement la forme d'équilibre prise par un massif d'enrochements jetés dans la houle. Ceri permet en particulier des observations intéressantes sur l'équilibre d'une jetée à ses diverses phases de construction.
Dans une deuxième série d'essais, nous avons soumis à diverses houles un massif de blocs en vrac ou d'enrochements, construit préalablement en eau calme. La forme d'écuilibre une fois obtenue, nous en avons mesuré les dimensions les plus caractéristiques.

Cette méthode permet d'étudier la stabilité do blocs ou d'enrochements d'une maniere plus générale cue par l'étude d'ouvrages particuliers.

Nous avons, pour terminer, comparé aux formules déià existantes le résultat de nos essaic. et essayé de dégager l'influence d'un certain nombre de paramètres.

\section{II. - ETUDE QUALITATIVE DU PROFIL D'EQUILIBRE D'UNE JETEE EN ENROCHEMENTS}

\section{A. - Ouvrage réalisé en enrochements homogènes}

Nous avons utilisé, au cours de cette première étude, des graviers de densité bien définie et dont le poids variait très peu d'un enrochement à l'autre.

Le talus naturel en eau calme de ce matériou était voisin de $4 / 3$.

Les essais, ainsi que ceux décrits plus Ioin, ont été effectués dans plusieurs canáx à honff. dont les caractéristiques générales ćtaient:

- longueur : $30 \mathrm{~m}$;

- largeur : $0,60 \mathrm{~m}$;

- profondeur maximum utilisée au cours des essais : $0,60 \mathrm{~m}$.

Ces canaux étaient équipés de l'appareillage nécessaire à la reproduction des houles aux caractéristiques les plus variées : volet batteur mû par un ensemble moteur électrique - variateur de vitesse - réducteur de vitesse - bielle manivelle à excentricité variable. Un jeu de filtres régularisait la houle et absorbait les réflexions parasites.

Les canaux sont constitués sur plusieurs mètres de leur longueur par des glaces verticales. au droit desquelles on peut observer facilement l'évolution des ouvrages et en photographier les phases les plus marquantes.

Les enrochements ont été jetés lentement à partir d'une trémie qui occupa pendant tout l'essai une position invariable. La houle gardait une période et une amplitude constantes.

On constate alors que l'on peut mettre en évi- dence trois phases caractéristiques parfaitement définies :

- Une phase d'exhaussement du massif, qui s'élève peu à peu dans les profondeurs où la houle n'a qu'une action minime,

Une phase d'allongement pendant laquelle le massif s'étire vers l'aval sans gagner beaucoup de hauteur, en « usant » peu à peut l'énergie incidente de la houle,

- Une phase d'émersion où le massif émerge ef ou la coupure se fait entre le large el la zone abritée.

\section{Premiere phase : Exhaussement (fig. 1).}

Il se forme au début un massif prismatioue centré sur l'axe de la trémie et qui ne differe pratiquement pas de celui que l'on obtiendrait en eau calme.

Cette phase d'exhaussement pur se poursuit jusqu'à ce que le massif submergé atteigne une profondeur limite à partix de laquelle les enrochements commencent à osciller. Cette profondeur, qui semble bien définie, dépend évidemment de la houle et des enrochements utilisés.

Si l'on poursuit l'expérience, le massif continue à s'élever peu à peu, son sommet, initialement arrondi, s'aplatissant encore.

A son passage sur l'ouvrage, la houle se modifie profondément : ́pour une hauteur suffisante du massif, sa crête donne naissance à 2 ou 3 vagues ou même plus.

Vers la fin de cette phase, le massif a conservé une section triangulaire, mais on peut constater 


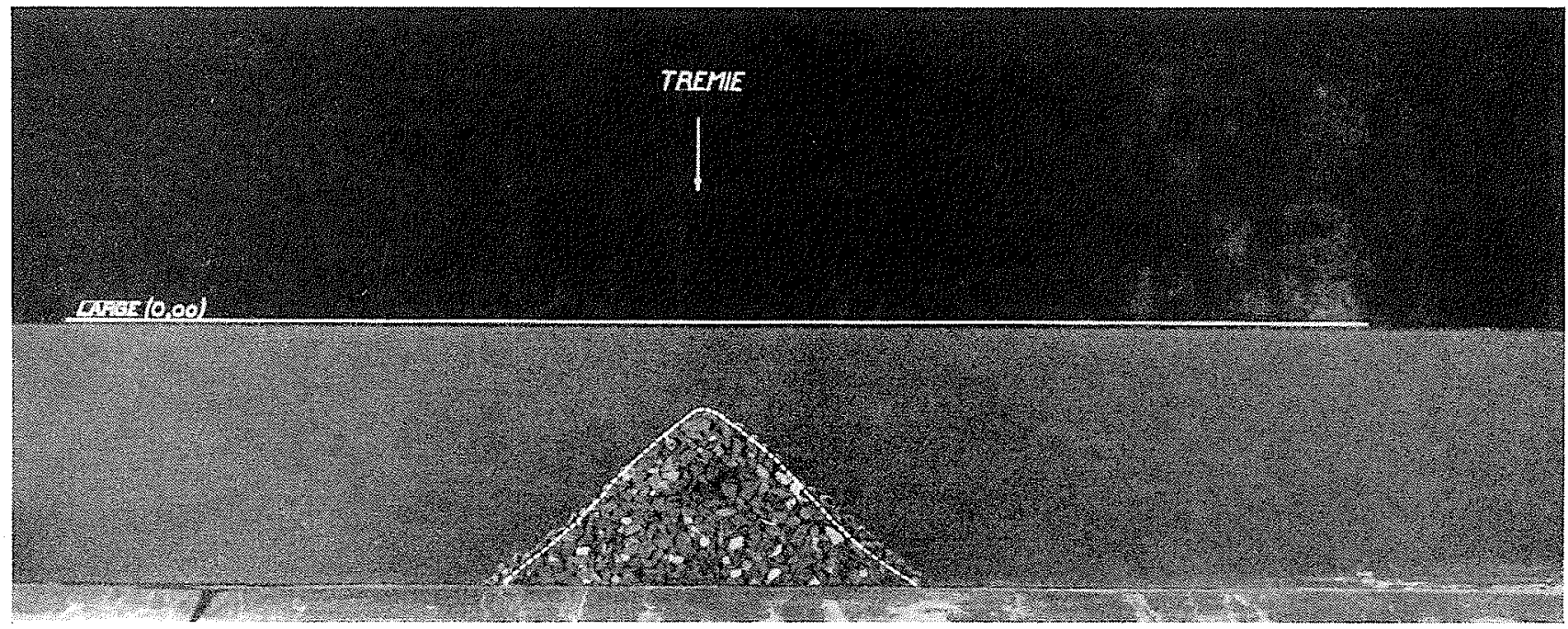

Fir. 1. - Phase d'exhaussement.

que son sommet s'est un peu déplacé dans le scns de propagation de la houle.

\section{Deuxiène phase : Allongement}

L'expérience se poursuivant lentement, la crête du massif atteint à un moment donné la profondeur de déferlement. Dès cet instant, l'évolution du massif change totalement.

Chaque bloc que l'on jette est alternativement sommis au courant qui accompagne le déferlement, puis au courant de retour et décrit ainsi un va-et-vient régulier à la période de la houle. Toutefois, le courant créé par le déferlement est plus violent que le courant de retour et l'enrochement se déplace peu à peu dans le sens de la houle pour venir finalement s'ébouler sur lit partie interne de la jetée.

L'évolution verticale de l'ouvrage marque un net temps d'arrêt et le massif progresse peu à peu en s'allongeant lentement et en prenant une forme sensiblement trapézoïdale caractéristique (fig. 2).

La « partie active 》du massif, c'est-à-dire le plateau supérieur en pente douce sur lequel la houle dissipe son énergie, s'élève lentement en. gardant une pente constante.

La houle à l'arrière de l'ouvrage diminue peu a peu; il est cependant nécessaire de monter le massif très près de la surface pour que son « effet amortisseur $\gg$ atteigne des valeurs vraiment intéressantes.

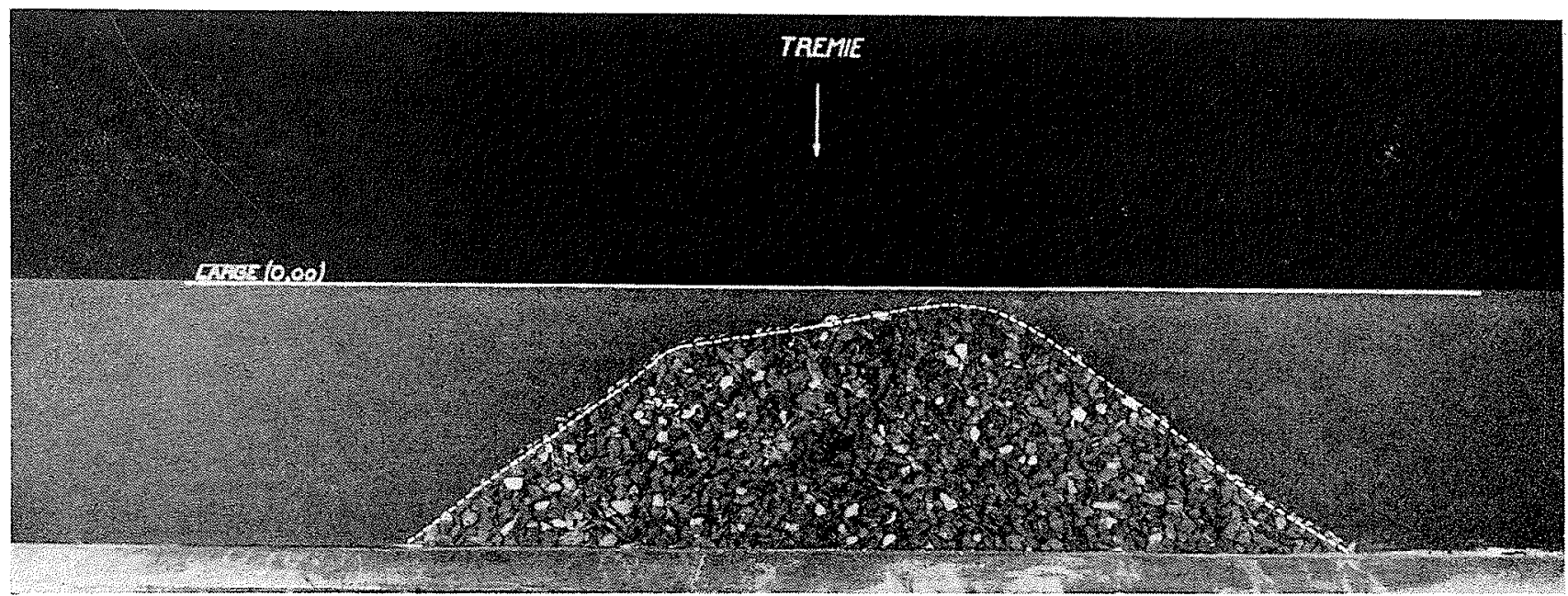

Fig. 2. - Phase d'allongement. 


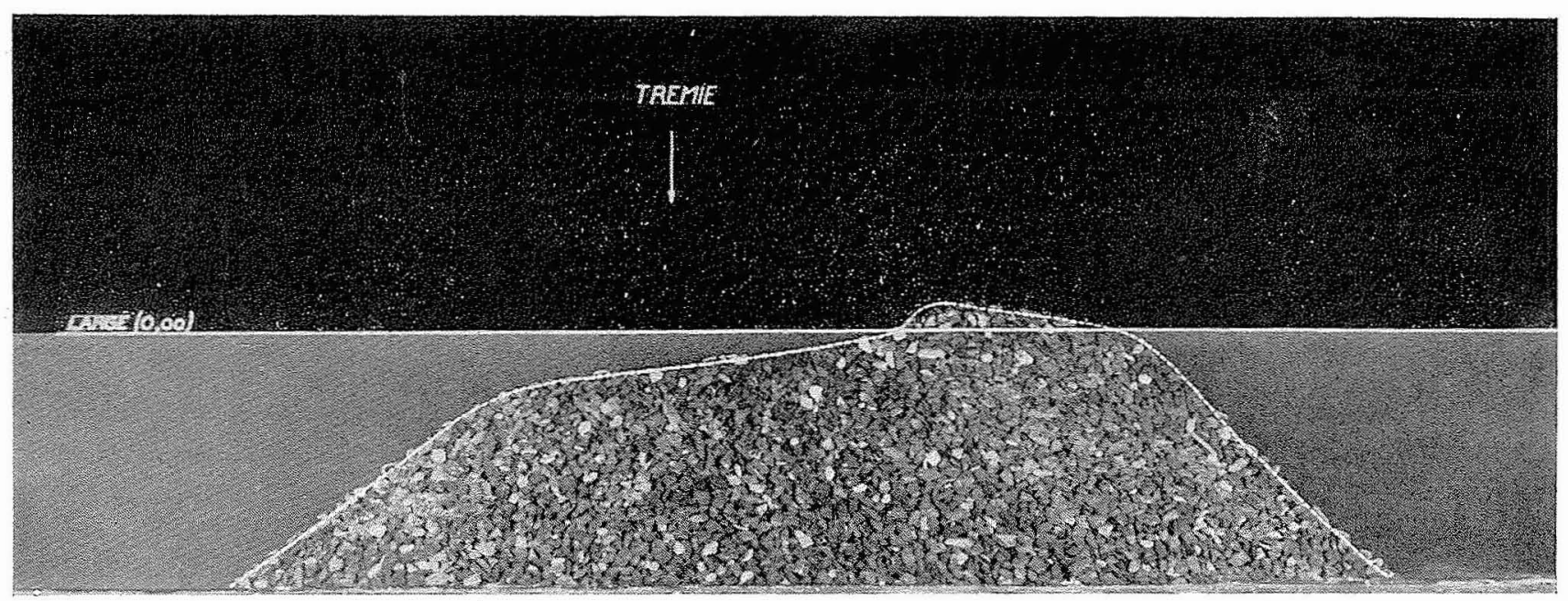

Fic. 3. - Phase d'émersion.

Troisì̀me phase: Emersion (fig. 3).

A partir d'une certaine cote, le sommet du massif émerge périodiquement et s'oppose à l'écoulement de retour de la zone abritée vers le large. Les enrochements qui, jusque-là, subissaient un mouvement alternatif, ne sont plus alors soumis qu'au seul jet de déferlement. L'évolution s'accélère et l'émersion se produit, très rapide par rapport à la lente succession des phases précédentes. Sur le trapèze de la figure 2 se greffe un petit massif triangulaire concave vers le large. Sa crête, que la houle ne franchit plus, indique le point le plus haut où elle est capable de soulever l'un quelconque des enrochements.
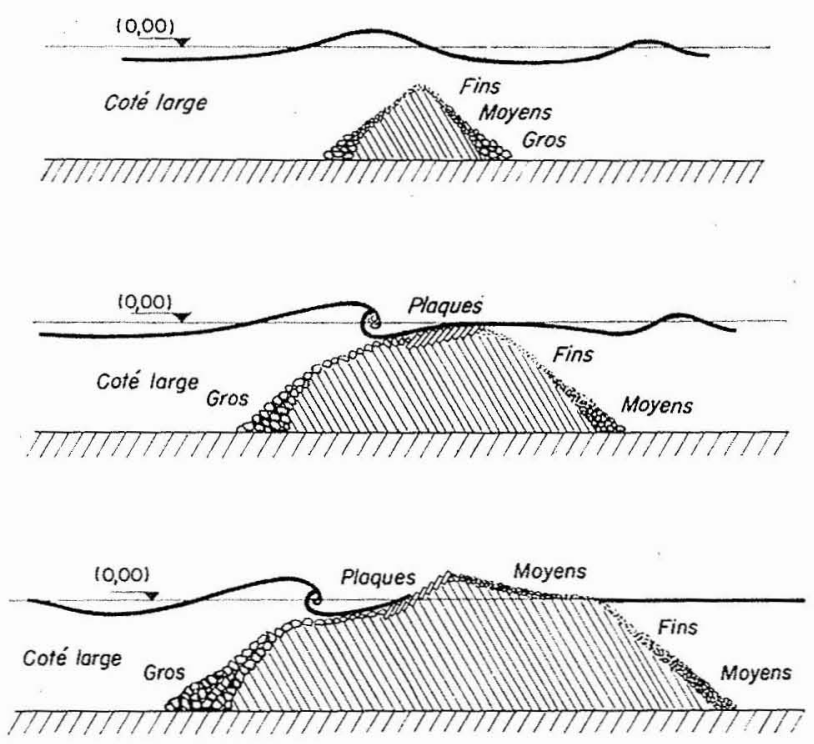

Fici 4. - Triage d'enrochements hétérogènes par la houle.
Si l'on poursuit le jet des blocs, le massif prismatique d'émersion gagne peu à peu vers le large sur le plateau supérieur qui n'évolue plus. Les pentes se raidissent, la crête du massif émergé s'élève au fur et à mesure que celui-ci s'avance vers le large.

Au bout de peu de temps, on obtient un profil qui présente les pentes les plus fortes compatibles avec la stabilité des enrochements vis-àvis de la houle considérée. Si l'on poursuit l'expérience, on voit le massif se déplacer en bloc parallèlement à lui-même vers le large en conservant les mêmes pentes.

Cette étude, faite avec un matériau homogène, a été complétée par une recherche, analogue, effectuée avec un matériau hétérogène, dans le but d'observer les lois du triage naturel qui président à la constitution du massif.

\section{B. - Ouvrage réalisé en enrochements hétérogènes}

Pour étudier le triage naturel, nous avons employé un mélange tout-venant de galets de rivière, dont les caractéristiques générales étaient :

- Talus naturel en eau calme : $5 / 3$;

-C Poids unitaire : de quelques centigrammes (sable) à $40 \mathrm{~g}$;

- Porosité du massif : faible (30\% environ).

La jetée évolue en présentant des phases successives identiques à celles vues plus haut.

Nous indiquerons donc simplement les lois du triage naturel (fig. 4). 
PREMLiER PHASE : EXHAUSSLMENT.

Pendant la phase d'exhaussement, le triage sur le massif prismatique est analogue à celui que l'on observe à sec ou en cau calme. Les gros blocs, qui ne trouvent que peu d'appuis à leur taille, s'éboulent jusqu'au pied des talus, alors que les moyens constituent les parties hautes de l'ouvrage et que les fins s'incrustent peu à peu dans les interstices.

\section{Deunieme phase : Allongement.}

Un tri s'effectue sur le plateau supérieur, directement soumis au déferlement. Les trés gros enrochements, quoique relativement stables, sont déchaussés peu à peu par Ia houle et finissent en général par rouler jusqu'au pied du talus cóté large. Quelques-uns résistent et recouvrent la partie inférieure du plateau d'une lourde carapace très efficace.

Les éléments fins sont chassés vers le talus còté port, où ils s'éboulent et colmatent tous les vides du talus.

Les éléments de dimensions moyennes sont poussés par la houle et viennent également s'ébouler còté port. Toutefois les galets plats, dont la portance est grande, ont tendance à se plaquer sur le plateau et finissent par en recouvrir la partie supérieure d'une carapace de larges plaques imbriquées comme les tuiles d'un toit, avec un pendage vers le large. Ce phénomène ne devient toutefois net qu'à partir du moment où le massif est suffisamment élevé pour que le courant de retour provenant, à chaque période, de la zone abritée, soit devenu presque négligeable.
Troistìme phase : EMLRSION.

Au moment de l'émersion, le triage ne change pas. On remarque simplement que les ćléments de dimensions moyennes ne peuvent plus gagner le talus coté port et demeurent sur la plage supérieure où ils roulent indéfiniment.

A la fin de l'expérience, le massif est done recouvert superficiellement, du large ver's le port : d'abord par un talus de très gros éléments, qui épaule le plateau supérieur recouvert d'une carapace de gros blocs. Au-delà, le plateau est recouvert par les plaques imbriquées et le prisme émergé est lui-même balayé par des élèments moyens sur sa face còté large. Le talus còté port est constitué par des éléments mélangés qui vont du moyen au très fin, avec quelques gros en pied.

Les éléments fins tendent à s'infiltrer par gravité dans les régions basses, qu'ils finissent par colmater completement. Dans les ouvrages reets on ne trouve que peu de fins, surtout à l'origine. Mais peu à peu, du fait de l'usure des enrochements de l'ouvrage d'une part et de l'apport en suspension par la houle, des sables et limons d'autre part, ces éléments s'accroissent et doivent venir se déposer peu à peu en colmatant les parties basses de l'ouvrage. Du point de vue de la porosité et de l'étanchement des jetées, il $y$ a done là un phénomène de vieillissement qu'll $y$ avait lieu de signaler.

Remarquons pour terminer que, si ce triage naturel est assez intéressant puisqu'il recouvre le platcau actif d'une carapace de blocs lourds et de plaques imbriquées, il est cependant très peu économique en ce sens que les éléments les plus gros, donc à la fois les plus cher's et les plus utiles pour la stabilité de l'ouvrage, tombent au pied des talus dans les régions profondes où ils ne servent que de remblai.

\section{III. - ÉTUDE QUANTITATIVE DU PROFIL D'ÉQUILIBRE D'UNE JETÉE EN BLOCS EN VRAC OU EN ENROCHEMENTS}

\section{A. - Profil d'équilibre d'une jetée en blocs en vrac}

Cette étude a été effectuće à la demande et pour le compte de la Direction du Port de Marseille et de la Chambre de Commerce de Marseille.

\section{a) Les maténiatx uTilisés.}

Au cours de la première série d'essais quantitatifs que nous allons exposer, nous avons utilisé des blocs en béton dont l'agrégat et le dosage étaient bien définis. Ces matériaux offraient l'avantage de posséder une densité régulière, ainsi que des formes simples, susceptibles d'être reproduites à volonté dans l'avenir.

Pour chaque densité étudiée, Ies essais furent menés successivement avec des blocs cubiques, des blocs cubiques semi-arrondis, des blocs cubiques arrondis (fig. 5). Ces déux dernières formes étaient obtenues en usant les arêtes des blocs cubiques pendant un temps convenable, réglé empiriquement, dans une bétonnière en rotation. 


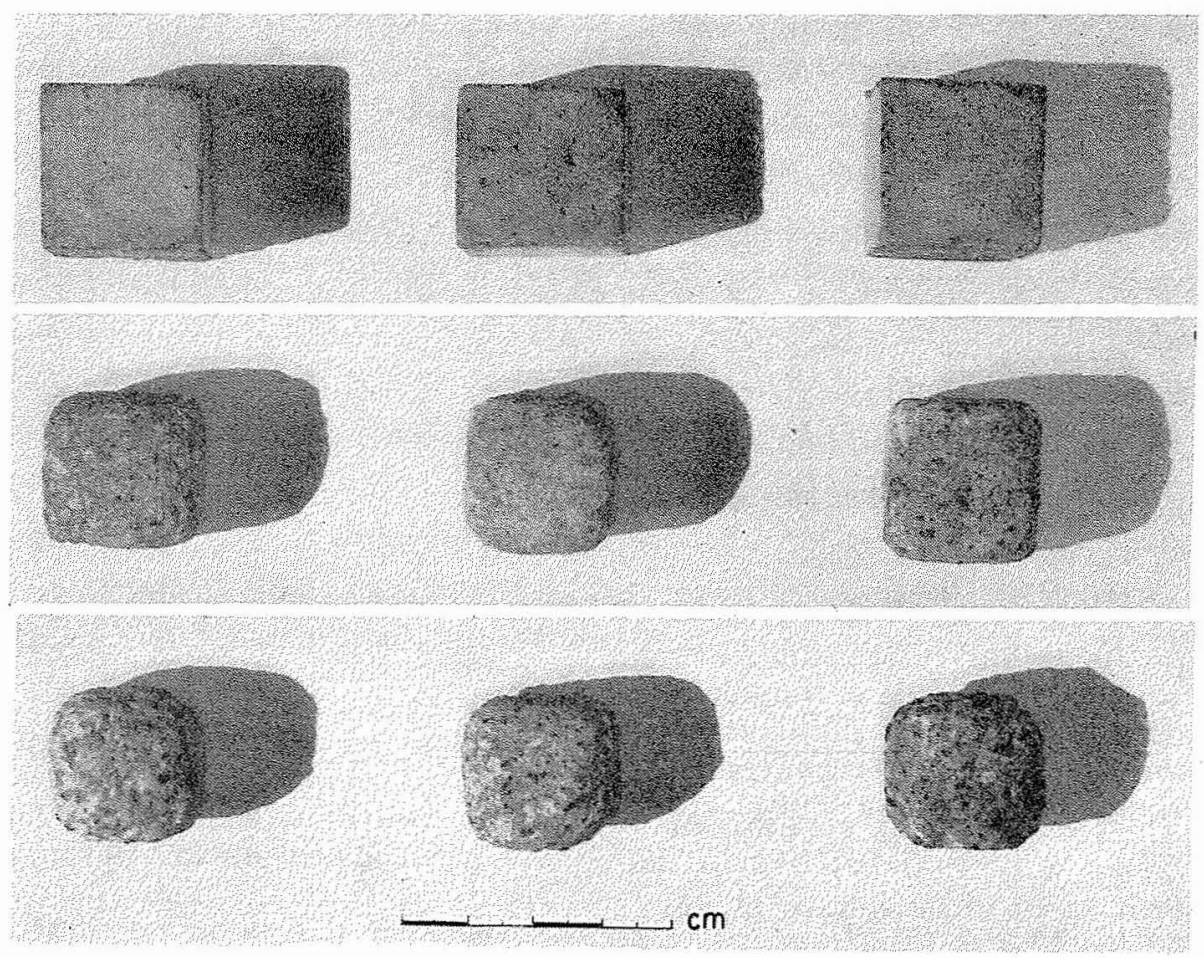

FIG. 5.

Blocs eubiques.

Bloes semi-arrondis.

Blocs arrondis. Densité 3,22.

Les caractéristiques des blocs utilisés sont portées dans le tableau ci-dessous :

\begin{tabular}{|c|c|c|c|}
\hline \multirow{2}{*}{$\begin{array}{c}\text { Densité } \\
\text { humide }\end{array}$} & \multicolumn{3}{|c|}{ Poids unitaire moyen des blocs en $\mathrm{g}$} \\
\cline { 2 - 5 } & cubiques & semi-arrondis & arrondis \\
\hline 1,87 & 30,70 & 29,20 & 20,80 \\
\hline 2,93 & 34,73 & 33,50 & 27,55 \\
\hline 3,22 & 40,00 & 33,50 & 24,50 \\
\hline
\end{tabular}

\section{b) LA TEGHNIQUE DES ESSAIS.}

Les blocs sont immergés dans l'eau calme, de manière à réaliser un massif prismatique limité par deux talus d'équilibre naturel. On crée ensuite une houle dont on mesure la période et l'amplitude devant l'ouvrage.

Attaquant la digue, cette houle y creuse peu à peu soǹ profil d'équilibre. Tantôt un bloc est soulevé par l'action combinée de l'effort direct de la vague et des forces dues aux sous-pressions, tantôt il est emporté par le courant de retour qui suit le déferlement; des pans entiers de l'ouvrage s'éboulent, sont déblayés par la houle et viennent remblayer la partie inférieure du talus. L'évolution, d'abord rapide, se ralentit peu à peu et on arrive enfin à un état d'équilibre.

La forme du talus d'équilibre à la houle ainsi obtenu peut être assimilée sans grande erreur à une ligne brisée (fig. 6). Parcourant le profil depuis le large jusqu'au côté abrité, on rencontre successivement :

- Un talus incliné à l'angle de talus naturel du matériau considéré;

- Le plateau supérieur, directement soumis au déferlement et qui fait avec l'horizontale un angle $\alpha$, variable dans chaque cas, et qui caractérise la stabilité à la houle du matériau utilisé;

-- Un talus émergent, très souvent plus incliné que le talus naturel du matériau; on sait en effet qu'en dégarnissant le pied d'un talus, ou obtient des pentes d'équilibre légèrement plus raides que celles obtenues en chargeant en crête; la houle procède ici d'une façon analogue; 
- Enfin, côté port, un talus incliné à l'angle d'équilibre naturel.

Ce profil en ligne brisée se retrouve dans ses grandes lignes quels que soient le matériau et la houle incidente. Seuls varient l'angle $\approx$ que fait avec l'horizontale le plateau supérieur et la profondeur de l'extrémité inférieure de celui-ci. Sa partie supérieure, où il se raccorde au talus raide émergé, est toujours située aux alentours du niveau $(0,00)$.

Quelques essais comparatifs nous ont montré que la valeur de $\alpha$ déterminée en jetant les blocs dans la houle, ainsi que nous l'avions fait au cours de l'étude qualitative, était très voisine de celle obtenue en faisant agir la houle sur un talus primitivement réglé à sa pente d'équilibre naturel. Nous avons donc adopté cette dernière méthode, plus rapide.

\section{c) Les Résultats.}

Nous présenterons les résultats de cette étude au paragraphe IV. Notons simplement ici que l'angle $\alpha$ défini plus haut est celui qui correspond à la stabilité générale du profil; un talus réglé à cet angle d'équilibre ne verra pas sa pente modifiée par la houle. Mais sur ce talus, stable, certains blocs peuvent être instables, osciller ou mème se déplacer d'un mouvement alternatif à la période de la houle.

Indépendamment de l'usure qu'il crée, ce mouvement des blocs est nuisible, car il peut engendrer un déplacement d'ensemble "en chevrons» lorsque la houle attaque obliquement la digue. Celle-ci se dégrade alors par transport Iongitu- dinal. Pour obtenir un profil dont tous les éléments soient stables, il conviendrait d'augmenter le poids individuel des blocs.

La profondeur de l'extrémité inférieure du plateau (qui par exemple sur la figure 6 s'établit aux environs de $-6,00$ ), est approximativement égale à la profondeur de déferlement de la houle : 1,3 à 1,4 fois l'amplitude au large.

\section{B. - Profil d'équilibre d'une jetée en enrochements (Fig. 6)}

Les essais précédents ayant porté sur des blocs cubiques bruts, émoussés ou arrondis, nous avons cherché si des enrochements naturels obéissaient aux mêmes lois, en dépit de la dispersion de leurs poids, densité et forme.

Nous avons donc effectué plusieurs séries d'essais sur des enrochements répondant aux caractéristiques suivantes:

$$
\begin{aligned}
& d=2,80 \text { - Poids moyen }=66 \mathrm{~g}-33 \mathrm{~g}-16,5 \mathrm{~g} \\
& 9,5 \mathrm{~g}-4 \mathrm{~g}-2 \mathrm{~g} \\
& \mathrm{~d}=2,60 \text { - Poids moyen }=35 \mathrm{~g} \\
& d=2,55 \text { - Poids moyen }=81,4 \mathrm{~g} \\
& \begin{aligned}
d=2,35 \text { - Poids moyen }=66 \mathrm{~g}-33 \mathrm{~g} \\
-16,5 \mathrm{~g}-8,75 \mathrm{~g}-3,55 \mathrm{~g}
\end{aligned}
\end{aligned}
$$

Ces divers enrochements ont été soumis à des houles d'amplitude et de période variables et la profondeur d'eau au pied de l'ouvrage a, elle aussi, varié au cours des essais.

Les résultats de ces études seront exposés également au paragraphe IV.

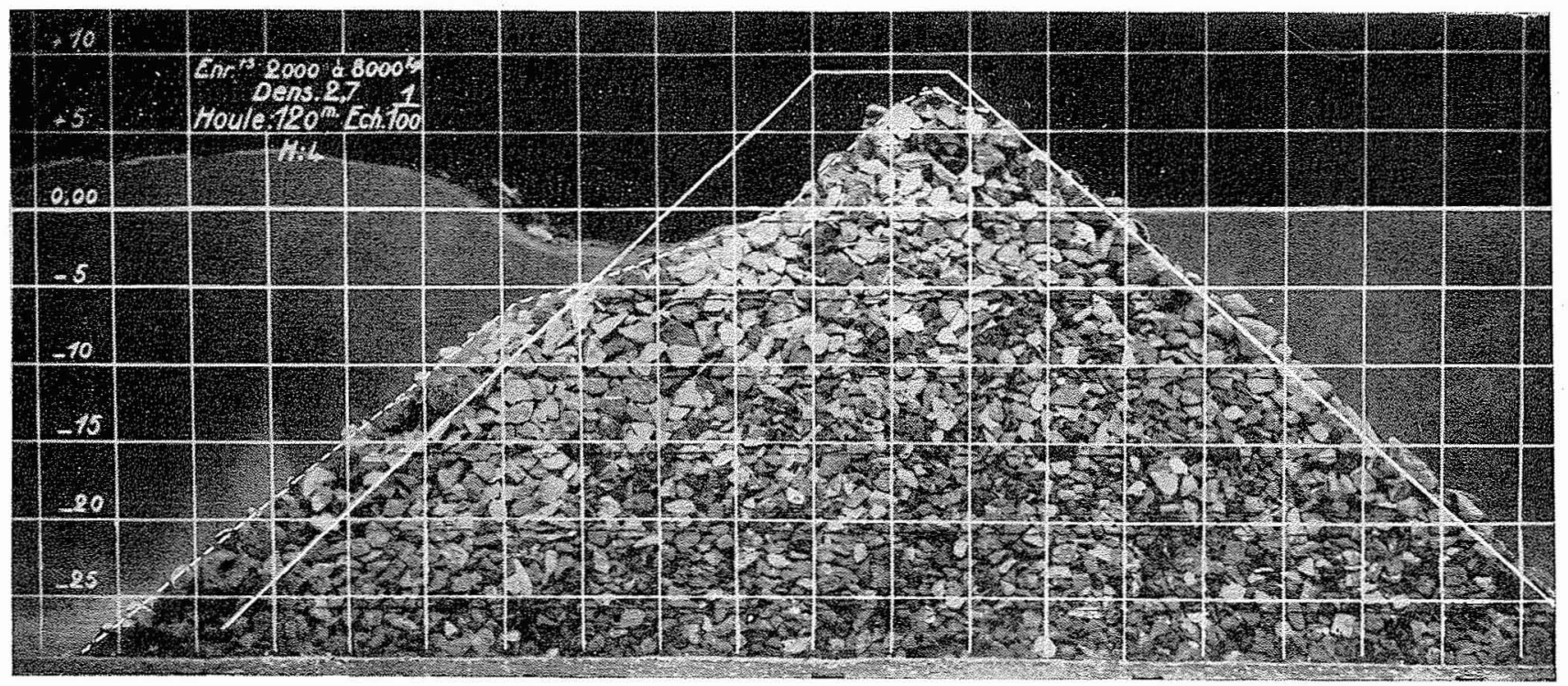

Fic. 6. - Profil d'équilibre d'une jetée en enrochements. 


\section{IV. - COMPARAISON ENTRE CES RÉSULTATS EXPÉRIMENTAUX ET LES VALEURS DÉDUITES DE DIFFÉRENTES FORMULES}

\section{A. - Formules de stabilité}

Il existe un certain nombre de formules it coefficients empiriques donnant la valeur de l'angle d'équilibre $\alpha$ en fonction des différents paramètres. Il n'existe pas à notre connaissance de formule déduite des théories mathématiques de la houle et donnant un résultat directement exploitable par le praticien.

Rappelons ici les notations employées.

$\alpha=$ angle d'équilibre à la houle du matériau considéré, défini plus haut;

$\mathrm{T}=$ période de la houle, en secondes;

$h=$ profondeur au pied de l'ouvrage, en mètres;

$2 a=$ amplitude de la houle à la profondeur $h$, en mètres;

$\mathrm{L}=$ = longueur d'onde de la houle à la profondeur $h$, en mètres;

$2 a_{0}=$ amplitude de la houle en profondeur infinie, en mètres;

$\mathrm{L}_{0}=$ longueur d'onde de la houle en profondeur infinie, en mètres;

$\mathrm{P}=$ poids des blocs, en tomnes;

$d=$ densité des blocs.

a) Formule de M. Inibarren [1]

$$
\mathrm{P}=\frac{\mathrm{K} \cdot 2 a_{d^{3}} \cdot d}{(d-1)^{3}(\cos \alpha-\sin \alpha)^{3}}
$$

où: :

$2 a_{d}=$ amplitude de la houle lors du déferlement; cette amplitude peut être calculée selon la méthode exposée par M. InrBARREN dans ses articles [2];

$\mathrm{K}=0,015$ pour des enrochements naturels;

$\mathrm{K}=0,019$ pour des blocs artificiels.

Dans le cas où la profondeur au pied de l'ouvrage est inférieure à $0,06 \mathrm{~L}_{0}$, on peut, d'après l'auteur [2] appliquer la formule:

$$
\mathrm{P}=\frac{\mathrm{N} \cdot 2 a^{3} \cdot d}{(d-1)^{3}(\cos \alpha-\sin \alpha)^{3}}
$$

où :

$\mathrm{N}=0,023$ pour des enrochements naturels;

$\mathrm{N}=0,029$ pour des blocs artificiels.
L'auteur recommande de multiplier les résultats obtenus pour $\mathrm{P}$ par un coefficient de sécurité convenable.

b) Formule de MM. Epstein et Tyrrell [4]

où :

$$
\mathrm{P}=\mathrm{R}_{t} \frac{2 a^{3} d}{(d-1)^{3}(\mu-\operatorname{tg} \alpha)^{3}}
$$

$R_{t}$ est une fonction de $\alpha, \mu$ et $h / L$, qui fait intervenir quatre coefficients inconnus;

$\mu=$ coefficient de frottement pierre sur pierre, peu différent de l'unité.

c) Formule de M. Castro [1]

$$
\mathrm{P}=\frac{0,7042 a^{3} d}{(\operatorname{cotg} \alpha+1)^{2}(d-1)^{3} \sqrt{\operatorname{cotg} \alpha-\frac{2}{d}}}
$$

d) Formule de M. Mathews [5]

$$
\mathrm{W}_{1}=\frac{6 w \mathrm{H}^{2} \mathrm{~T}}{(w-64)^{3}(\cos \alpha-0,75 \sin \alpha)^{2}}
$$

où :

$W_{1}=\underset{\text { poids de l'enrochement en tonnes de } 2.000}{\text { livres; }}$

$w=$ poids spécifique des enrochements en livres par pied cube;

$\mathrm{H}=$ amplitude de la houle en pieds.

formule que nous pouvons transcrire sous une forme semblable aux précédentes, avec les mêmes notations :

$$
\mathrm{P}=\frac{0,001492 a^{2} \mathrm{~T} d}{(\cos \alpha-0,75 \sin \alpha)^{2}(d-1)^{3}}
$$

e) Formule de M. RodolH [5]

$$
\mathrm{W}_{1}=\frac{\mathrm{H}^{2} \mathrm{~T} d}{600 \operatorname{tg}^{3}\left(45^{\circ}-\frac{\alpha}{2}\right)(d-1)^{3}}
$$

oll encore :

$$
\mathrm{P}=\frac{0,01622 \alpha^{2} \mathrm{~T} d}{\operatorname{tg}^{3}\left(45^{\circ}-\frac{\alpha}{2}\right) \cdot(d-1)^{3}}
$$


f) Formule de M. LaRras [6]

$$
\begin{gathered}
\sin \left(45^{\circ}-\alpha\right)=\frac{\mathrm{K} \cdot 2 a_{0} \cdot d^{1 / 3}}{\mathrm{P}^{1 / 3}(d-1)}\left(\frac{4 \pi a_{0} / \mathrm{L}}{\operatorname{sh} 4 \pi \mathrm{H} / \mathrm{L}}\right) \\
k=0,175 \text { pour des enrochements, } \\
k=0,189 \text { pour des blocs artificiels. }
\end{gathered}
$$

Transcrite sous une forme semblable aux précédentes, cette formule devient :

$$
\mathrm{P}=\frac{\mathrm{K} \cdot\left(2 a_{0}\right)^{3} d}{(d-1)^{3}(\cos \alpha-\sin \alpha)^{3}}\left(\frac{4 \pi a_{0} / \mathrm{L}}{\operatorname{sh} 4 \pi \mathrm{H} / \mathrm{L}}\right)^{3}
$$

où :

$\mathrm{K}=0,0152$ pour des enrochements naturels;

$\mathrm{K}=0,0191$ pour des blocs artificiels;

$\mathrm{H}=$ profondeur du point considéré.

Il convient « de faire toujours au moins $\mathrm{H}=a_{0}$ dans la formule précédente, même quand $\mathrm{H}$ est en réalité plus faible, puisqu'on est alors en plein dans la lame déferlante ».

Comparons les valeurs données par ces diverses formules aux résultats des essais précédemments décrits, en présentant l'influence des différents paramètres sur le poids $P$.

\section{B. - Influence de la densité des éléments}

Tous les auteurs précédents, à l'exception de M. Castro, admettent que l'influence de la densité se traduit par le terme $d /(d-1)^{3}$. Ceci ré- sulte, ainsi qu'un calcul simple le montre, de la prise en compte de la poussée d'Archimède. On peut se demander si cette prise en compte est bien justifiée, étant donné la complexité du problème. Admettre que c'est le poids propre du bloc et non son poids apparent qui intervient, conduit à une loi de la forme $1 / d^{2}$ au lieu de $d /(d-1)^{8}$.

Nous avons tracé (fig. 7) la variation de $\mathbf{P}$ en fonction de $d$ suivant ces deux lois (les autres paramètres restant constants) et porté la moyenne de nos points expérimentaux. On voit que la loi $d /(d-1)^{3}$ donne des résultats plus proches de la réalité que la forme $1 / d^{2}$.

Cependant la loi $d /(d-1)^{3}$ ne semble pas parfaitement acceptable. La dispersion des points expérimentaux, ainsi que le faible nombre de densités étudiées, ne nous permet pas de conclure de façon plus précise.

Si l'on tient compte de ce que la densité $d_{0}$ de l'eau de mer est différente de l'unité, $\mathbf{P}$ varie alor's comme :

$$
\frac{d}{\left(\frac{d-a_{0}}{d_{0}}\right)^{3}}
$$

$d_{0}$ étant voisin de 1,03 , on est ainsi conduit à majorer $\mathrm{P}$ de 10 à $15 \%$.

\section{C. - Influence de la forme des éléments}

Les blocs arrondis utilisés au cour's des essais se sont révélés moins stables que les cubes à arêtes vives, les blocs semi-arrondis ayant une stabilité intermédiaire.

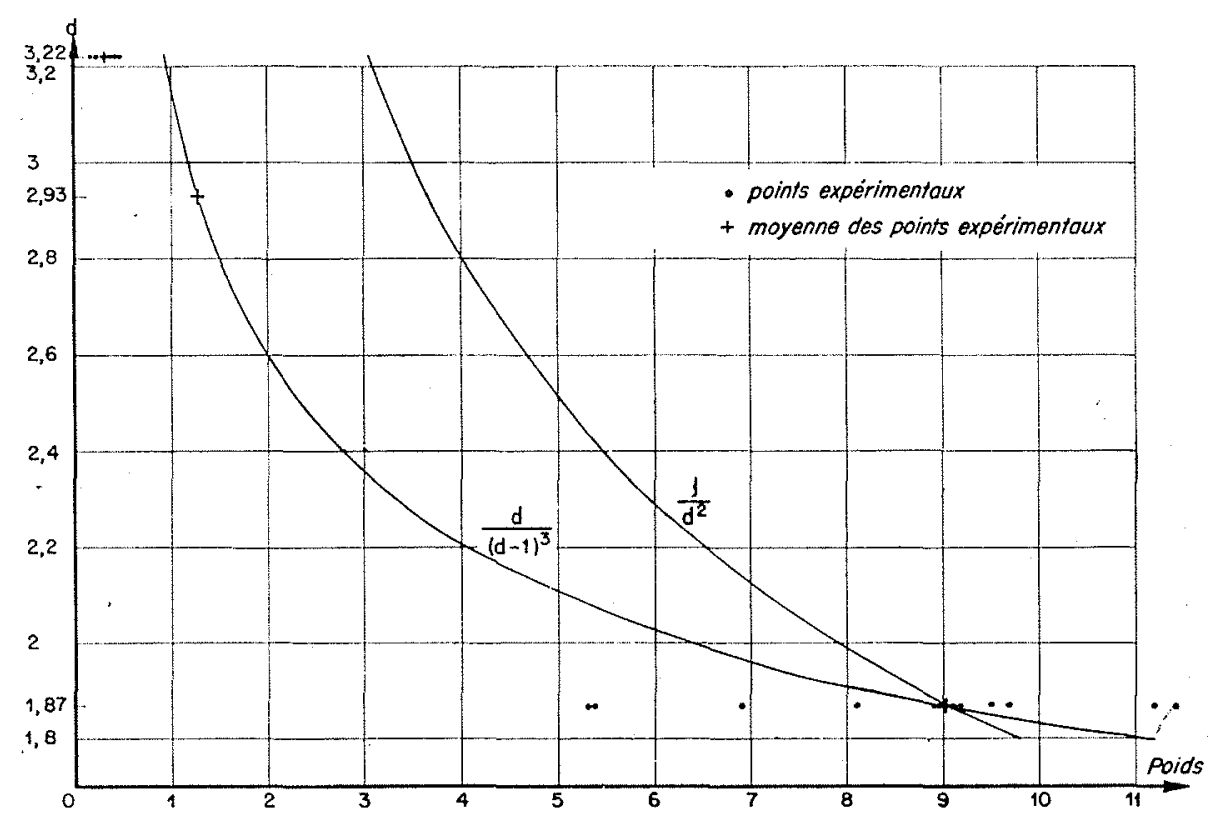

Frc. 7. - Influence de la densité des éléments. 
Pour obtenir une stabilité égale à celle de blocs cubiques de poids $P$, il convient d'employer des blocs semi arrondis de poids 1,35 $\mathrm{P}$ ou des blocs arrondis pesant $3 \mathrm{P}$.

Dans le cas d'une jetée dont la carapace est formée de blocs artificiels, on voit que l'usure (inévitable) des arêtes peut, au cours des années, causer des dommages importants. Il est nécessaire de tenir compte de cette usure dans la détermination du poids des blocs.

En ce qui concerne les enrochements naturels, une difficulté se présente : préciser leur poids. Si nous le définissons comme la moyenne arithmétique des poids de tous les enrochements composant la catégorie, l'enrochement de stabilité égale à celle du bloc cubique de poids $P$ devra peser $3 \mathrm{P}$ - toutes choses égales par ailleurs, en particulier la densité. Nous pensons cependant que la forme même des enrochements doit avoir un rôle nọ négligeable. Notons que ces essais ont porté sur des enrochements émoussés par rotation dans une bétonnière. Dans la pratique des travaux à la mer, les enrochements bénéficient souvent d'une densité supérieure à celle du béton, ce qui améliore nettement leur stabilité.

\section{D. - Influence de la profondeur au pied de l'ouvrage}

Certains essais ont été effectués à des profondeurs variables entre $3 a$ et $12 a$.

Si l'on prend comme amplitude de référence celle existant à l'aplomb du pied de la jetée, on ne peut mettre en évidence une influence de la profondeur; celle-ci, si elle existe, est masquée par la dispersion des mesures.

\section{E. - Influence de la période et de l'amplitude de la houle}

Nous n'avons pu, de même, déceler une influence de la période, dissimulée, elle aussi, si elle existe, par la dispersion des résultats.

En ce qui concerne l'amplitude, les formules existantes admettent généralement une loi $\mathrm{P}=\mathrm{K} \times 2 a^{3}$, homogène. Remarquons que les formules de Mathews et de Rodolph, qui font exception, conduisent, dans la pratique, à une variation similaire du poids en fonction de l'amplitude. Au cours d'une tempête, en effet, l'amplitude et la période varient généralement dans le même sens, ainsi qu'en font foi de nombreuses observations. Le terme $2 \alpha^{2} \mathrm{~T}$ varie alors dans le même sens que $2 a^{3}$.

\section{F. - Influence de la pente d'équilibre}

(Fig. 8 et 9)

C'est ici que divergent nettement les formules précédentes. Pour les comparer de façon précise, nous avons porté pour chacune le groupement $\mathrm{P} /\left(2 a^{*} d\right)$, sans dimensions, en fonction

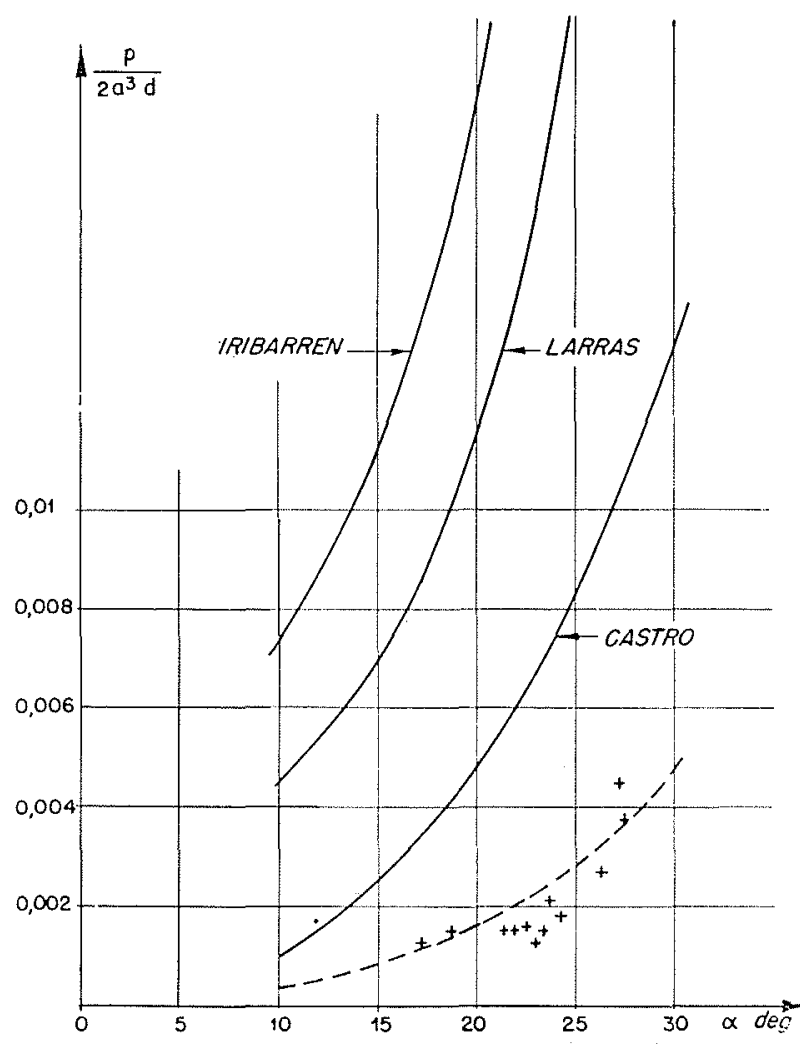

Fia 8 . - Stabilité de blocs cubiques.

$$
d=2,93 \quad \mathrm{P}=34,7 \mathrm{~g}
$$

de $\alpha$, et comparé aux résultats expérimentaux, pour des blocs cubiques $(d=2,93)$ et pour des enrochements $(d=2,35)$.

Cette méthode de comparaison n'offrait pas de difficultés pour la formule de MM. IrIBArren et Castro.

En ce qui concerne la formule de M. Larras, nous avons pris :

$$
\frac{4 \pi a_{0} / \mathrm{L}}{\operatorname{sh} 4 \pi a_{0} / \mathrm{L}}=0,975
$$

valeur moyenne au cours des essais.

Les formules de MM. Mathews et Rodolph n'étant pas homogènes, ne peuvent être portées sur ce tableau de façon précise. 


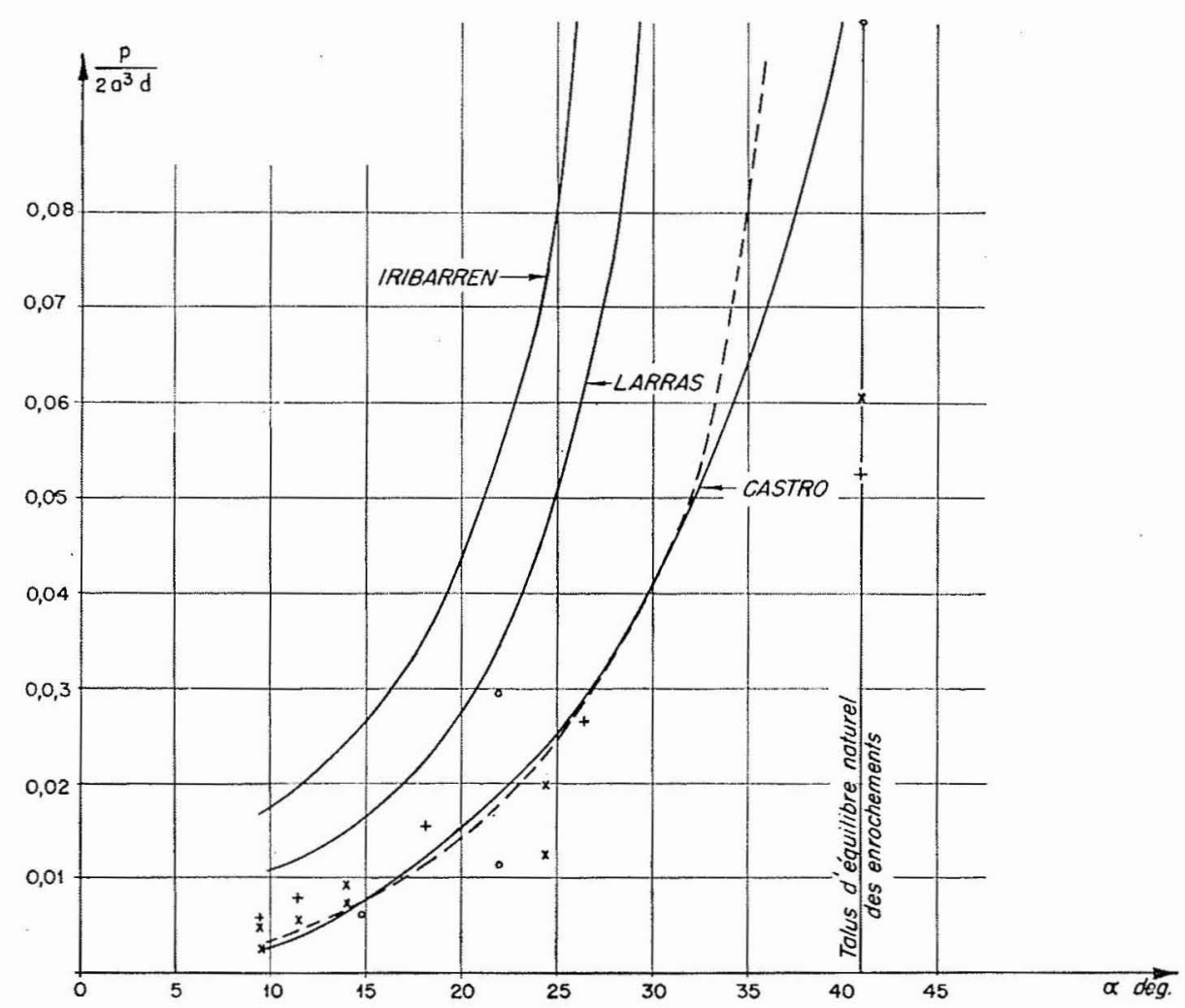

FIG. 9. - Stabilité d'enrochements.

$$
d=2,35 \quad \mathrm{P}=8,75 \mathrm{~g}
$$

Nous n'avons représenté que deux séries de points expérimentaux (cubes $d=2,93$, enrochements $d=2,35$ ). Les conclusions que l'on peut tirer de l'examen de ces figures sont confirmées par lous les autres essais effectués, que nous avons jugé inutile de reproduire ici. En effet, les nombreux facteurs en jeu font que nos points expérimentaux ne peuvent être portés sur un seul graphique. Reproduire l'ensemble de nos résultats nous aurait conduit à insérer un grand nombre de tableaux.

Un talus d'éléments en vrac réglé à sa pente d'équilibre naturel en eau calme peut-il résister sans dommage à des houles d'amplitude non négligeable? Cette question est importante, car l'Ingénieur cherchera toujours à raidir la pente des talus, pour des raisons évidentes d'économie. A cet égard, les formules précédentes se rangent en deux catégories :

- Celles de MM. Iribarren, Epstein et TyrREL et LARRAS conduisent à adopter des blocs très lourds pour former des talus d'équilibre naturel (voisins de 4/3). Pour un talus théorique à $1 / 1$, le poids des éléments deviendrait infini.

- Les formules de MM. Castro, Mathriws et RoDOLPH, au contraire, conduisent à des poids nettement moins importants pour des talus voi- sins de $4 / 3$ et à des blocs de poids fini pour un talus de $1 / 1$.

Nos essais semblent montrer que c'est cette dernière hypothèse qui est conforme à la réalité : un talus d'équilibre naturel peut résister sans dommages à des houles d'amplitude finie.

M. Iribarren, ainsi que MM. Epstein et TyrREL, pensent en effet que la chute d'un enrochement à la surface du talus se produit par glissement, ce qui les conduit à admettre qu'un talus d'équilibre en eau calme est à la limite de sa stabilité et qu'il ne peut résister à aucune houle si faible soit-elle. En réalité, l'observation sur modèle réduit montre que les chutes se produisent par roulement des enrochements sur les blocs sous-jacents. Un calcul simple, que nous ne reproduirons pas ici, démontre alors qu'un talus naturel est en équilibre stable et peut résister à des houles d'amplitude non négligeable.

Pour en terminer avec l'influence de la pente du talus, notons que l'attaque de la houle s'effectue de façon notablement différente suivant la valeur de cette pente :

- Pour de faibles valeurs de $\alpha$, il se produit, même pour des houles peu cambrées, un déferlement qui provoque une destruction brutale de l'énergie et une érosion violente. 
- Pour des valeurs de a proches de l'angle de talus naturel, la houle au contraire se réfléchit notablement sur l'ouvrage, tant que l'on n'atteint pas des cambrures déjà importantes. C'est dans ce cas par éboulements dus aux variations de pression statique que le massif tend vers sa pente d'équilibre et non sous l'effet des efforts dynamiques des lames.

Les appuis des blocs les uns sur les autres sont également différents. Plus le talus est raide, plus les blocs se coincent mutuellement.

Ces deux facteurs font qu'une même formule semble pouvoir difficilement s'appliquer à tous les talus, quelle que soit leur pente.

\section{G. - Abaque de stabilité}

Nous avons porté sur un graphique (fig. 10) le résultat moyen des essais effectués jusqu'à ce jour au Laboratoire Dauphinois d'Hydraulique. En fonction de $\alpha$ et de $d$, cet abaque donne la valeur de $\mathrm{P} / 2 a^{3}$; connaissant $2 a$, on peut donc déterminer le poids minimum $\mathrm{P}$ de stabilité sous l'angle $\alpha$, en eau douce. Ce graphique est valable pour des blocs cubiques ou pour des enrochements naturels.

Nous avons figuré en pointillés sur les figures $\delta$ et 9 les courbes déduites de l'abaque de stabilité (fig. 10). On voit qu'elles donnent bien une valeur moyenne des résultats expérimentaux.

\section{V. - CONCLUSION}

L'abaque de la figure 10 peut servir à la détermination approchée des éléments constituant la carapace d'une digue à talus.

Il convient de multiplier alor's la valeur de $\mathrm{P}$ indiquée par l'abaque par un coefficient de sécurité convenable. Celui-ci reste à définir par la pratique; il semble qu'il ne doive pas être inférieur à 2,5 .

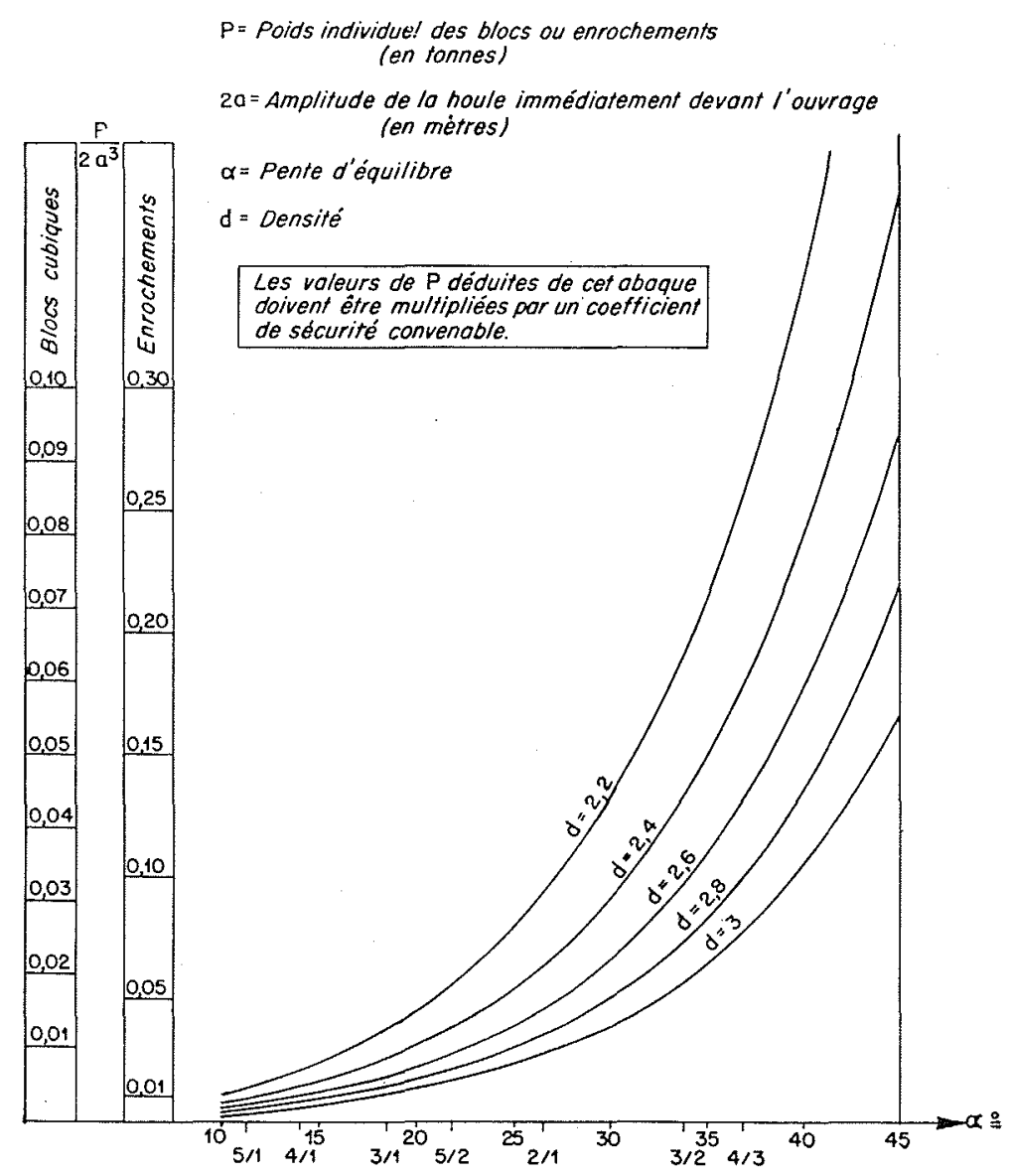

FIG. 10. - Pente d'équilibre. 
Nous voudrions en effet attirer l'attention de l'utilisateur éventuel sur les nombreuses causes d'indétermination qui subsistent. Il faut noter que :

- Ce graphique donne les valeurs moyennes des résultats expérimentaux. Or, ceux-ci présentent une dispersion très importante, donnant parfois des valeurs de $\mathrm{P}$ variant du simple au double.

- La forme d'une digue classique s'écarte nettement de celle du massif homogène étudié, en particulier par la présence d'une superstructure souvent non poreuse.

- Nous avons négligé l'influence apparemment faible de la période et de la profondeur au pied de l'ouvrage; ces facteurs pourraient, dans certains cas, prendre éventuellement de l'importance.

- Les enrochements présentent un échelonnement des poids et des densités qui se prête mal à la définition de $\mathrm{P}$ et $d$.

- Nous avons simplifié l'étude en considérant un problème « à deux dimensions ». Dans la réalité, une attaque oblique de la houle pourrait peut-être conduire à d'autres résultats.

- Nos essais ont été effectués en eau douce. La densité plus forte de l'cau de mer conduit, nous l'avons vu, à des valeurs de $\mathrm{P}$ plus grandes.

- Le passage du modèle réduit à la réalité peut comporter une certaine indétermination si l'échelle correspondante est trop faible.

- L'amplitude maximum à redouter est le plus souvent mal connue, il convient de ne la faire intervenir dans les calculs que multiplée elle-même par un coefficient de sécurité convenable.

Ces réserves indispensables font que la détermination a priori de la stabilité d'un talus est toujours fort hasardeuse. II suffit pour le cons- tater de comparer les écarts existant entre les formules proposées. Celles-ci, ainsi que notro graphique, ne doivent être utilisées que pour chiflrer un avant-projet, elles ne dispensent pas d'une étude plus approfondic, ni d'essais particuliers sur modèle réduit.

Cet abaque doit être considéré comme provisoire, de nouvelles études pouvant nous amener à compléter les données déjà recueillies, en particulier en ce qui concerne l'influence de la densité. Nous avons pensé que, malgré ses lacunes, il pourrait être utile aux utilisateurs. Leur expérience des Travaux Maritimes doit d'ailleurs leur permettre de nous aider à définir un coefficient de sécurité qui complète de façon pratique les résultats déjà obtenus. Nous les en remercions par avance.

\section{BIBLIOGRA PHIE}

(1) IndBarren. - Bulletin of the Beach Erosion Board, janvier 1949. p. 1.

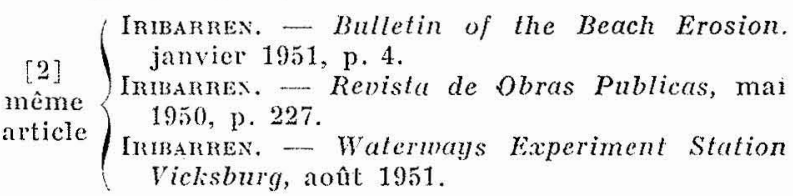

[3] Ayuso. - Dock and Marbour, septembre 1946, p. 117 .

[1] Epstein et Truel.t. - XVII Congrès International de Navigation, Lisbonne. $\mathrm{S}_{2} \mathrm{C}_{4}$. p. 81 .

[5] Hikson et Ronolph. - Coastal engeneering, octobre 1950, p. 227.

[6] Larnas. - Génie civil, 15 septembre 1952, p. 353.

[7] Robert Y. Hunson. - Proceedings. American Society of Civil Engineers, janvier 1952, vol. 78, separate n" 113.

[8] K. Kaptan. R.G. Hennes, C.E. Leonoff et R. Hudson. Proceedings. American Society of Civil Engineers. Discussion, voi. 78 , septembre 1953 .

[9] VEsper et K. Kaplax. - Bulletin of the Beach Erosion Board, janvier 1953 , vol. $7, \mathrm{n}^{\circ} 1$.

[10] Inubarren. - Revista de obras publicas, janvier 1953 , p. 12. 